University of Nebraska - Lincoln

DigitalCommons@University of Nebraska - Lincoln

\title{
Carbon 1s Photoelectron Spectroscopy of Halomethanes. Effects of Electronegativity, Hardness, Charge Distribution, and Relaxation
}

\author{
T. Darrah Thomas \\ Oregon State University, T.Darrah.Thomas@oregonstate.edu \\ Leif J. Saethre \\ University of Bergen \\ Knut J. Børve \\ University of Bergen \\ John D. Bozek \\ University of California \\ Marko Huttula \\ University of Oulu \\ See next page for additional authors
}

Follow this and additional works at: https://digitalcommons.unl.edu/usdoepub

Part of the Bioresource and Agricultural Engineering Commons

Thomas, T. Darrah; Saethre, Leif J.; Børve, Knut J.; Bozek, John D.; Huttula, Marko; and Kukk, Edwin, "Carbon 1s Photoelectron Spectroscopy of Halomethanes. Effects of Electronegativity, Hardness, Charge Distribution, and Relaxation" (2004). US Department of Energy Publications. 44.

https://digitalcommons.unl.edu/usdoepub/44

This Article is brought to you for free and open access by the U.S. Department of Energy at DigitalCommons@University of Nebraska - Lincoln. It has been accepted for inclusion in US Department of Energy Publications by an authorized administrator of DigitalCommons@University of Nebraska - Lincoln. 


\section{Authors}

T. Darrah Thomas, Leif J. Saethre, Knut J. Børve, John D. Bozek, Marko Huttula, and Edwin Kukk 


\title{
Carbon 1s Photoelectron Spectroscopy of Halomethanes. Effects of Electronegativity, Hardness, Charge Distribution, and Relaxation
}

\author{
T. Darrah Thomas, ${ }^{* \dagger}$ Leif J. Saethre, ${ }^{*}, *$ Knut J. Børve,,$* \star *$ John D. Bozek, ${ }^{\perp}$ \\ Marko Huttula, ${ }^{\S}$ and Edwin Kukk ${ }^{\S}$ \\ Department of Chemistry, Oregon State University, Corvallis, Oregon 97331-4003, Department of Chemistry, \\ University of Bergen, NO-5007 Bergen, Norway, Advanced Light Source, Lawrence Berkeley Laboratory, \\ University of California, Berkeley, California 94720, and Department of Physical Sciences, University of Oulu, \\ FIN-90014, Oulu, Finland
}

Received: February 3, 2004; In Final Form: March 31, 2004

\begin{abstract}
Carbon 1s ionization energies have been measured for 12 halomethanes. These together with earlier measurements provide 27 compounds for investigating the relationship between core-ionization energies and the electronegativity and hardness of the halogens. The ionization energies correlate nearly linearly with the sum of the electronegativities of the halogens attached to the central carbon. Both electronegativity and hardness play important roles in determining the ionization energy, and it is found that the linear relationship between ionization energy and electronegativity arises from an interplay of the electronegativity and hardness of the halogens and the length and ionicity of the carbon-halogen bond.
\end{abstract}

\section{Introduction}

The concept of electronegativity - the ability of an atom (or functional group) to attract electrons to itself-plays an important role in chemistry. A related concept is the hardness, which has been defined to be half the derivative of electronegativity with respect to charge. ${ }^{1}$ It is inversely related to polarizability. ${ }^{2,3} \mathrm{~A}$ number of quantitative definitions of electronegativity have been given and a number of tables of electronegativity and hardness have been presented. Despite this extensive activity the quantitative nature of both of these remains elusive. In particular, different definitions of electronegativity lead to different results and, in some cases, to different ordering of the electronegativity of common elements. With regard to hardness, there is limited quantitative experimental information.

Moreover, the concept of a single electronegativity for an element is not adequate: the electronegativities of $=\mathrm{O}$ and $-\mathrm{OH}$ are quite different, as are the electronegativities of $-\mathrm{CH}_{2} \mathrm{CH}_{3}$ and $-\mathrm{CH}=\mathrm{CH}_{2}$. Considerable effort-theoretical and experimental-has gone into attempts to assign group electronegativities, but these efforts have not been entirely successful. A tool that can provide insight into group electronegativity and hardness for a variety of groups would be useful.

Inner-shell ionization energies reflect both the charge distribution in a molecule (and, hence, the electronegativity of its component atoms) and the polarizability of the molecule (and, hence, the hardness of the component atoms). It is not surprising, therefore, that the core-ionization energies of a central atom correlate with the electronegativities of the substituents attached to the atom. It has been our goal to use these correlations to obtain a better insight into the nature of electronegativity and to develop a method for assigning group electronegativities on the basis of core-ionization energies.

* To whom correspondence should be addressed. E-mail: darrah.thomas@orst.edu; leif.saethre@kj.uib.no; knut.borve@kj.uib.no.

$\dagger$ Oregon State University.

University of Bergen.

$\perp$ University of California.

$\S$ University of Oulu.
The halomethanes provide a useful set of base compounds for this purpose. They all have essentially the same structure and bonding, but the electronegativities and polarizabilities of the halogens vary over a wide range. Carbon 1 s ionization energies have been reported for 17 halomethanes. We present here the results of new measurements for two of these $\left(\mathrm{CH}_{2} \mathrm{~F}_{2}\right.$ and $\mathrm{CH}_{3} \mathrm{Br}$ ) plus measurements for ten additional halomethanes $\left(\mathrm{CH}_{2} \mathrm{Br}_{2}, \mathrm{CHBr}_{3}, \mathrm{CH}_{2} \mathrm{I}_{2}, \mathrm{CH}_{2} \mathrm{ClF}, \mathrm{CHClF}_{2}, \mathrm{CHCl}_{2} \mathrm{~F}, \mathrm{CFBr}_{3}\right.$, $\mathrm{CH}_{2} \mathrm{BrI}, \mathrm{CH}_{2} \mathrm{ClI}$, and $\mathrm{CH}_{2} \mathrm{BrI}$ ) chosen to show the systematic variation of the ionization energy with type and number of halogens.

The results are discussed in terms of first-principles calculations of the ionization energies, the contributions of electrostatic potential and polarizability to the ionization energies, and the relationship between the core-ionization energies and the electronegativity and hardness of the halogens.

\section{Experimental Procedures and Experimental Results}

The experimental measurements of carbon 1s ionization energies have been carried out at the Advanced Light Source, using beamline 10.0.1 and the HIRAMES facility, ${ }^{4}$ and at the MAX-lab, using beamline I411. ${ }^{5}$ In each case photons of 330 $\mathrm{eV}$ were used to ionize carbon. The photoelectrons were detected in a Scienta SES 200 analyzer. The combined monochromator and analyzer slits and the analyzer pass energy were chosen to give a resolution of $75 \mathrm{meV}$ in the photoelectron spectra. This was verified by measurements of the methane carbon $1 \mathrm{~s}$ photoelectron spectrum.

Calibration of the kinetic energy scale of the analyzer was based on measurements of the xenon $\mathrm{N}_{4,5} \mathrm{OO}$ Auger spectrum. ${ }^{6}$ Calibration of the ionization energy scale was obtained by running a calibrating gas $\left(\mathrm{CO}_{2}\right.$ or $\left.\mathrm{CF}_{4}\right)$ mixed with the sample of interest. The calibration is thus based on the known adiabatic ionization energies for $\mathrm{CO}_{2}$ and $\mathrm{CF}_{4}{ }^{7}$

The spectra have been fit by least squares with peak shapes that include the effects of resolution, lifetime, and the interaction of the photo and Auger electrons (post-collision interaction, or 


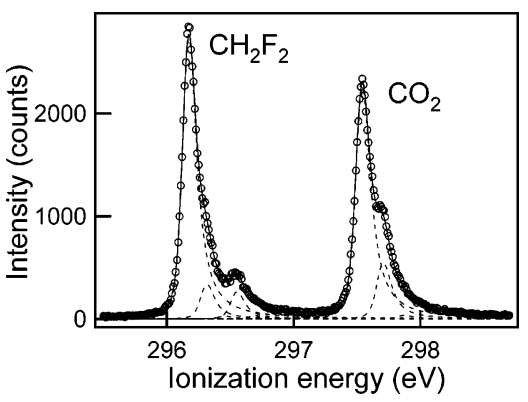

Figure 1. Carbon 1s photoelectron spectra for $\mathrm{CH}_{2} \mathrm{~F}_{2}$ and $\mathrm{CO}_{2}$.

PCI). ${ }^{8}$ The line shape given by eq 12 from van der Straten et al. ${ }^{9}$ was used to describe the effect of PCI. This was convoluted with a Gaussian function representing the effect of the experimental resolution. The resolution function was taken to have a fixed full width at half-maximum of $75 \mathrm{meV}$. The Lorentzian width, reflecting the lifetime of the core-hole state, was one of the fitting variables and was found to range from 89 to 103 $\mathrm{meV}$, with an average of $95 \mathrm{meV}$. This value is consistent with other values of the carbon 1s Lorentzian line width that we have measured. ${ }^{10}$ At the low end of this range are the widths for $\mathrm{CH}_{2} \mathrm{~F}_{2}$ and $\mathrm{CHF}_{2} \mathrm{Cl}$, both at $89 \mathrm{meV}$. These are intermediate between the typical values for hydrocarbons of 95 to $100 \mathrm{meV}$ and that of $\mathrm{CF}_{4}$, which is $77 \mathrm{meV} .{ }^{10}$

Each spectrum has a characteristic vibrational structure and this has been modeled by using one or two vibrational progressions. When hydrogen is present there is a noticeable $\mathrm{CH}$ stretching progression with a characteristic energy of about $0.4 \mathrm{eV}$. In addition, there are contributions from bending modes or lower frequency stretching modes with characteristic energies of about $0.1 \mathrm{eV}$; these are modeled with a single mode. In general, the spectra are dominated by the peak representing ionization to the vibrational ground state of the ion (adiabatic ionization), and the energy assigned to this peak by the fitting procedure is not sensitive to the details of the vibrational structure.

In Figure 1, the carbon 1s photoelectron spectrum of $\mathrm{CH}_{2} \mathrm{~F}_{2}$ together with the calibration spectrum for $\mathrm{CO}_{2}$ illustrates the features. In this figure, the open circles represent the experimental data, the solid line the overall least-squares fit, and the dashed lines the components of the fit. The peak at an ionization energy of about $296.6 \mathrm{eV}$ arises from the $v=1$ excitation of the $\mathrm{CH}$ stretch, and the peak that contributes to the shoulder on the main peak of the $\mathrm{CH}_{2} \mathrm{~F}_{2}$ spectrum is due to $\mathrm{CF}$ stretching and $\mathrm{HCH}$ bending. In the $\mathrm{CO}_{2}$ spectrum the relative energies and heights of the peaks have been constrained to the values measured previously. ${ }^{11}$ The Lorentzian widths for the two molecules were allowed to vary separately and were found to be $89 \mathrm{meV}$ for $\mathrm{CH}_{2} \mathrm{~F}_{2}$, as noted above, and $97 \mathrm{meV}$ for $\mathrm{CO}_{2}$, in agreement with earlier results. ${ }^{11}$

In Table 1 are listed the results of our measurements of the adiabatic and vertical ionization energies for 12 halomethanes. The adiabatic ionization energy is the energy needed to produce the ion in its vibrational ground state and for these molecules is obtained from the position of the main peak in the spectrum. The vertical ionization energy is obtained from the average over the entire spectrum. Older, low-resolution measurements give only the vertical energy. Also included here are the results of other measurements for 15 other halomethanes. ${ }^{7,12-16}$ For the data from the literature some modifications have been made to the reported results, reflecting changes in the values of reference energies. For instance, the ionization energies given by Perry and Jolly ${ }^{16}$ are, on the average, $0.13 \mathrm{eV}$ lower than the most
TABLE 1: Adiabatic and Vertical Ionization Energies for Halomethanes (eV)

\begin{tabular}{llllllll}
\hline & adiabatic & vertical & ref & & adiabatic & vertical & ref \\
\hline $\mathrm{CH}_{4}$ & 290.689 & 290.844 & $a$ & $\mathrm{CHF}_{2} \mathrm{Cl}$ & 297.671 & 297.700 & $b$ \\
$\mathrm{CH}_{3} \mathrm{~F}$ & 293.478 & 293.557 & $a$ & $\mathrm{CHFCl}_{2}$ & 296.334 & 296.372 & $b$ \\
$\mathrm{CH}_{3} \mathrm{Cl}$ & 292.29 & 292.43 & $c$ & $\mathrm{CHCl}_{3}$ & 295.12 & 295.16 & $c$ \\
$\mathrm{CH}_{3} \mathrm{Br}$ & 291.945 & 292.056 & $b$ & $\mathrm{CHBr}_{3}$ & 293.961 & 293.991 & $b$ \\
$\mathrm{CH}_{3} \mathrm{I}$ & & 291.43 & $d$ & $\mathrm{CF}_{4}$ & 301.898 & 301.898 & $a$ \\
$\mathrm{CH}_{2} \mathrm{~F}_{2}$ & 296.296 & 296.346 & $b$ & $\mathrm{CF}_{3} \mathrm{Cl}$ & & 300.25 & $e$ \\
$\mathrm{CH}_{2} \mathrm{FCl}$ & 294.977 & 295.036 & $b$ & $\mathrm{CF}_{3} \mathrm{Br}$ & & 299.77 & $f$ \\
$\mathrm{CH}_{2} \mathrm{Cl}$ & 293.73 & 293.81 & $c$ & $\mathrm{CF}_{3} \mathrm{I}$ & & 299.00 & $d$ \\
$\mathrm{CH}_{2} \mathrm{ClBr}$ & 293.39 & 293.456 & $b$ & $\mathrm{CF}_{2} \mathrm{Cl}_{2}$ & & 298.87 & $e$ \\
$\mathrm{CH}_{2} \mathrm{ClI}$ & 292.627 & 292.696 & $b$ & $\mathrm{CFCl}_{3}$ & & 294.48 & $e$ \\
$\mathrm{CH}_{2} \mathrm{Br}$ & 293.020 & 293.101 & $b$ & $\mathrm{CFBr}_{3}$ & 296.266 & 296.276 & $b$ \\
$\mathrm{CH}_{2} \mathrm{BrI}$ & 292.440 & 292.508 & $b$ & $\mathrm{CCl}_{4}$ & 296.32 & 296.33 & $c$ \\
$\mathrm{CH}_{2} \mathrm{I}_{2}$ & 291.924 & 291.983 & $b$ & $\mathrm{CBr}_{4}$ & & 294.77 & $g$ \\
$\mathrm{CHF}_{3}$ & 299.143 & 299.159 & $a$ & & & &
\end{tabular}

${ }^{a}$ Reference 7. ${ }^{b}$ This work. ${ }^{c}$ Reference $12 .{ }^{d}$ Reference $13 .{ }^{e}$ Reference 14. ${ }^{f}$ Estimated. See text. ${ }^{g}$ Reference 16.

recent values. Accordingly, the value listed for $\mathrm{CBr}_{4}$ in Table 1 is higher than they have reported by this amount. Similarly, the values given by Holmes ${ }^{14}$ for $\mathrm{CF}_{3} \mathrm{Cl}, \mathrm{CF}_{2} \mathrm{Cl}_{2}$, and $\mathrm{CFCl}_{3}$ have been decreased by $0.06 \mathrm{eV}$.

For $\mathrm{CF}_{3} \mathrm{Br}$, Jolly and Bakke $^{15}$ report that the carbon $1 \mathrm{~s}$ ionization energy is higher by $0.19 \mathrm{eV}$ than that of $\mathrm{CF}_{3} \mathrm{H}$. This difference is suspect, since replacing hydrogen by bromine typically leads to a shift of about $1.2 \mathrm{eV}$ in the carbon $1 \mathrm{~s}$ ionization energy. Although the shift is diminished by the presence of other halogen substituents, it is unlikely that it is as small as $0.19 \mathrm{eV}$. This value is discordant in the correlation discussed by Jolly and Bakke, and from the deviation of the point from the correlation line, we estimate that the reported shift is too low by about $0.5 \mathrm{eV}$. A more accurate estimate can be obtained by comparing the measured carbon $1 \mathrm{~s}$ ionization energies of $\mathrm{CF}_{3} \mathrm{I}, \mathrm{CF}_{3} \mathrm{Cl}$, and $\mathrm{CF}_{4}$ (relative to $\mathrm{CF}_{3} \mathrm{H}$ ) with those calculated theoretically; these are linearly related. From this relationship, together with the calculated ionization energy for $\mathrm{CF}_{3} \mathrm{Br}$, we estimate that this shift should be $0.62 \mathrm{eV}$, giving a value of 299.77 for the carbon 1s ionization energy of $\mathrm{CF}_{3} \mathrm{Br}$. We use this in our subsequent discussion and plan to remeasure it in the future.

The absolute uncertainties for our measurements (as well those of Myrseth et al. ${ }^{7}$ ) are about $0.03 \mathrm{eV}$ and the relative uncertainties are not more than about $0.02 \mathrm{eV}$. Since the relative uncertainties are unknown, we have retained 3 decimal places in reporting the results. For the other measurements, the uncertainties are reported to be between 0.05 and $0.07 \mathrm{eV}$.

\section{Theoretical Comparisons}

Theoretical ionization energies as well as other properties of the molecules discussed have been calculated with Gaussian98, ${ }^{17}$ using the B3LYP approach. For neutral molecules the aug-ccpVTZ basis set has been used for hydrogen, carbon, fluorine, and chlorine and the Stuttgart relativistic effective core potential (ECP) combined with the SDB-aug-cc-pVTZ basis set has been used for bromine and iodine. ${ }^{18-20}$ For core-ionized molecules, the scale factor for the carbon basis set was 0.964 and the core hole has been simulated with an effective core potential. The ECP used for this was that of Stevens et al. ${ }^{21}$ scaled to account for only one electron in the $1 \mathrm{~s}$ shell. ${ }^{22}$ For each molecule the optimized geometry of the ground state was determined, and this geometry was used in the calculations, using the ECP to represent the core hole.

The vertical ionization energy is the difference between the energy of the ground state and the energy of the core-ionized 
molecule having the same geometry as the ground state. The energy calculated with the ECP differs from the true energy of the core-ionized state, but we assume that this difference, which depends primarily on properties of the carbon core, is independent of the molecule. In this case, the difference will cancel out if we consider ionization energies relative to that of some standard molecule. We expect, therefore, that this approach will give reasonable predictions of the relative vertical ionization energies.

A comparison of theoretically calculated and experimentally measured relative ionization energies shows that the agreement is reasonably good but not perfect. The root-mean-square (rms) deviation between experiment and theory is $0.14 \mathrm{eV}$, which is more than 5 times the uncertainty in the relative measurements. The linear regression of the calculated and experimental results has a slope of 1.014, indicating that on the average the calculated shifts are slightly higher than the measured ones. The differences between the calculated and experimental values are, however, not random, but systematic. This can be seen by considering families of molecules, such as $\mathrm{CH}_{4-n} \mathrm{X}_{n}$, where $\mathrm{X}$ is one of the halogens. For $\mathrm{X}=\mathrm{F}$, the theoretical calculations overestimate the shifts by only $0.1 \%$, whereas for $\mathrm{X}=\mathrm{I}$, the corresponding number is $13 \%$. Chlorine and bromine fall between these extremes, at $3.9 \%$ and $5.4 \%$, respectively.

Overall, the agreement between theory and experiment is sufficiently good that we can with some confidence use the theoretical results in developing a better insight into the origin of the shifts in ionization energy that have been observed for these molecules. In the discussion below, we use these theoretical results together with the experimental ionization energies to assess the influence of initial-state charge distribution and final-state relaxation on the ionization energies.

\section{Systematics of the Ionization Energies}

Correlation with Number of Halogens. The experimental vertical carbon 1s ionization energies of the halomethanes relative to methane, $\Delta I$, can be fit reasonably well with an expression that is linear in the number of halogens of each type. A fit using such an expression gives $R^{2}$ equal to 0.996 with an rms deviation of $0.19 \mathrm{eV}$. It is to be noted, however, that the deviation is 5 to 10 times the relative uncertainty in the measurements, and this deviation reflects systematic deviation between the data and the linear relationship.

A better description is obtained by including quadratic terms, and, for this purpose, we use a multivariate second-order polynomial

$$
\Delta I=n_{\mathrm{X}} A_{\mathrm{X}}+n_{\mathrm{Y}} A_{\mathrm{Y}}+\frac{n_{\mathrm{X}}\left(n_{\mathrm{X}}-1\right)}{2} B_{\mathrm{XX}}+\frac{n_{\mathrm{Y}}\left(n_{\mathrm{Y}}-1\right)}{2} B_{\mathrm{YY}}+
$$

where $n_{\mathrm{X}}$ and $n_{\mathrm{Y}}$ are the numbers of halogens $\mathrm{X}$ and $\mathrm{Y}$. The linear coefficients are equal to the ionization-energy shift when one hydrogen atom is replaced by a halogen, and are expected to be related to the electronegativities of the halogens. The quadratic coefficients reflect how an added halogen modifies the influence of the other halogens. A regression with this expression gives $R^{2}$ equal to 0.99994 with an rms deviation of $0.03 \mathrm{eV}$. Thus, this expression describes the ionization energy within the experimental uncertainties.

The values of the coefficients are given in Table 2. Also listed here for comparison are the electronegativities of the halogens, ${ }^{23}$ and it can be seen that the values of $A_{\mathrm{X}}$ correlate well with these. This correlation is the subject of the following section.
TABLE 2: Coefficients of the Quadratic Correlation of Carbon 1s Ionization Energies $(\mathrm{eV})^{a}$

\begin{tabular}{lllll}
\hline & \multicolumn{1}{c}{$\mathrm{F}$} & \multicolumn{1}{c}{$\mathrm{Cl}$} & \multicolumn{1}{c}{$\mathrm{Br}$} & \multicolumn{1}{c}{$\mathrm{I}$} \\
\hline$A_{\mathrm{X}}$ & $2.758(16)$ & $1.569(16)$ & $1.199(18)$ & $0.59(3)$ \\
Pauling elctronegativity & & & & \\
$B_{\mathrm{XX}}$ & & & & \\
$B_{\mathrm{FX}}$ & $0.002(13)$ & $-0.134(13)$ & $-0.144(14)$ & $-0.03(7)$ \\
$C_{\mathrm{ClX}}$ & & $-0.132(9)$ & $-0.174(11)$ & $-0.236(17)$ \\
$C_{\mathrm{BrX}}$ & & & $-0.16(4)$ & $-0.30(4)$ \\
& & & & $-0.12(5)$
\end{tabular}

${ }^{a}$ The numbers in parentheses are the uncertainties in the last digit, as derived from the correlation. ${ }^{b}$ Reference 23 .

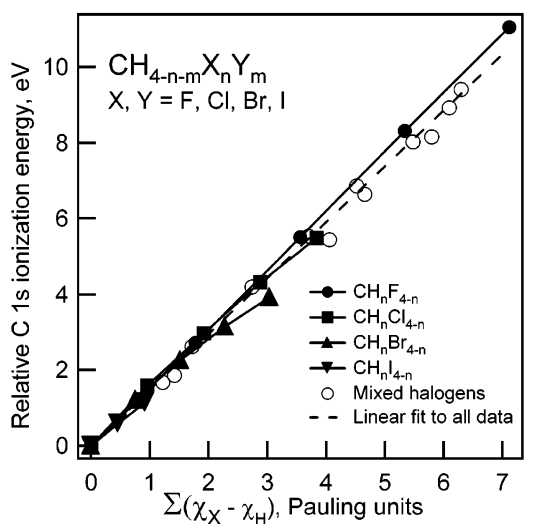

Figure 2. Experimental carbon 1s ionization energies in halomethanes plotted against the sum of the electronegativities of the halogens.

Correlation with Electronegativity. In 1970, Thomas showed that there was a linear correlation between the Pauling electronegativity of the halogens and the carbon $1 \mathrm{~s}$ core ionization energies of eight halomethanes. ${ }^{24}$ An expanded version of this correlation, including halomethanes, haloethanes, and haloethenes, has recently been presented by True et al. ${ }^{25}$ Specifically, the correlation is with the quantity $\sum\left(\chi_{\mathrm{X}}-\chi_{\mathrm{H}}\right)$, where $\chi_{\mathrm{X}}$ is the electronegativity of a ligand, $\chi_{\mathrm{H}}$ is the electronegativity of hydrogen, and the sum is taken over the ligands. With the additional measurements now available, it is possible to investigate this correlation in more detail.

An example of such a correlation is shown in Figure 2, where the experimental carbon 1s ionization energy of halomethanes (relative to that of methane) is plotted against $\sum\left(\chi_{\mathrm{X}}-\chi_{\mathrm{H}}\right)$, using the Pauling scale for the values of the electronegativities. ${ }^{23}$ The dashed line in Figure 2 shows a fit of a straight line to the data. The fit is reasonably good, with $R^{2}$ equal to 0.993 ; the rms deviation of the data from the fit line is $0.26 \mathrm{eV}$. This description of the results, with only one free parameter, is only slightly worse than the four-parameter linear fit described above. The slope of the line is $1.48 \mathrm{eV}$ per Pauling electronegativity unit, the same as found by Thomas $^{24}$ using a more limited set of data and by True et al. ${ }^{25}$ from halogenated methane, ethane, and ethene. The particular set of electronegativity values used here is only one of many that have been proposed. Several electronegativity scales ${ }^{26-29}$ give correlations similar to that shown, with the slope ranging from 1.4 to 1.6, whereas others $^{30-33}$ give rather poor correlations.

In Figure 2, data corresponding to families of compounds, $\mathrm{CH}_{n} \mathrm{X}_{4-n}(\mathrm{X}=\mathrm{F}, \mathrm{Cl}, \mathrm{Br}, \mathrm{I})$, are indicated by different symbols and connected by solid lines. We see that although the overall correlation is reasonably good in Figure 2, these families deviate from the straight line representing the fit. These deviations are related to the quadratic terms mentioned above.

The halogen electronegativity and the carbon $1 \mathrm{~s}$ ionization energy both reflect the ability of the halogen to remove electrons 


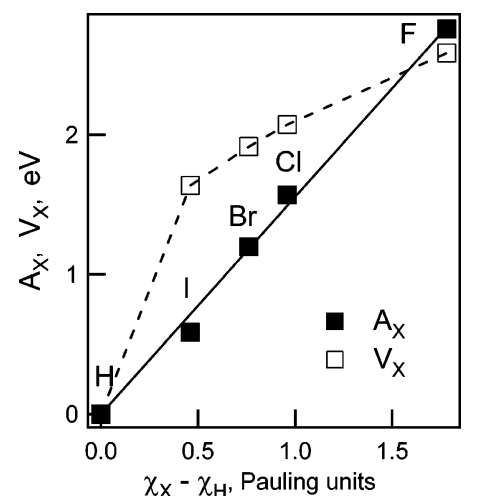

Figure 3. Linear coefficients $A_{\mathrm{X}}$ (solid) and $V_{\mathrm{X}}$ (open) plotted against substituent electronegativity. The solid line shows a least-squares fit to the data. The dashed line connects the points.

from the carbon atom, and it is, therefore, not surprising that the ionization energies increase with the number of halogens. It is also reasonable that this increase should be approximately linear. However, it is by no means obvious that the coefficient relating the ionization energy to the electronegativity (1.48) should be the same for all of the halogens. Another way to think about this question is to consider how the linear coefficients $A_{\mathrm{X}}$ vary with the electronegativity of the halogen. We might well expect $A_{\mathrm{X}}$ to increase monotonically with electronegativity, but there is no a priori reason to expect it to vary linearly. However, this is indeed the case, as can be seen from Figure 3, where the solid squares show $A_{\mathrm{X}}$ versus the electronegativity difference. The solid line represents a least-squares fit of a straight line to the points, and the linear relationship between $A_{\mathrm{X}}$ and electronegativity is apparent. We will return to this rather surprising result after discussing in the next section the factors that influence the shifts in core-ionization energies.

Initial State and Relaxation Effects. In simplest approximation (Koopmans theorem) the ionization energy is the negative of the energy of the orbital from which the electron is removed. This approximation is incorrect in that it neglects correlation effects as well as the response (relaxation) of the spectator electrons to the removal of the ionized electrons. For core electrons the relaxation effect is usually more important than the correlation effect, and, as a result, it has been convenient to describe core-ionization-energy shifts with two terms, $\Delta I=\Delta V$ $-\Delta R$, where $V$ reflects the effect of the initial-state properties of the molecule on the ionization energy and $R$ the effect of relaxation. $R$ is indicated with a negative sign since its effect is to make the ionization energy less than it would be if there were no relaxation.

In the past, $\Delta V$ has been equated either to $-\Delta \epsilon$ or to $\Delta U$, where $\epsilon$ is the orbital energy and $U$ is the potential energy of a unit positive charge at the center of the core-ionized atom. Although approximately correct, neither of these approaches is strictly correct, and we use instead the extended Koopmans theorem suggested by Børve and Thomas, ${ }^{34} \Delta V^{\mathrm{EKT}} \approx-\Delta \epsilon+$ $\Delta U^{\mathrm{VCI}}-\Delta U^{\mathrm{HF}}$, where $U^{\mathrm{VCI}}$ is the potential energy calculated with a valence-correlated level of theory and $U^{\mathrm{HF}}$ is the potential energy calculated at the Hartree-Fock level. In practice, $\Delta \epsilon$, $\Delta U^{\mathrm{VCI}}$, and $\Delta U^{\mathrm{HF}}$ are comparable in magnitude and approximately proportional to one another, so that the observations that follow depend only in detail on which of these quantities we use. The quantities $V, U$, and $\epsilon$ reflect primarily the Coulomb interaction of the core electron with the rest of the charges in the molecule and, consequently, $\Delta V, \Delta U$, and $\Delta \epsilon$ reflect differences in the charge distribution between the molecule of interest and the reference molecule.
TABLE 3: Relative Ionization Energies (I), Initial-State Energies $\left(V^{\mathrm{EKT}}\right)$, and Relaxation Energies $(R)(\mathrm{All}$ in $\mathrm{eV})$

\begin{tabular}{lllllrrr}
\hline & $\Delta I$ & $\Delta V^{\text {EKT }}$ & \multicolumn{1}{c}{$\Delta R$} & & \multicolumn{1}{c}{$\Delta I$} & $\Delta V^{\text {EKT }}$ & \multicolumn{1}{c}{$\Delta R$} \\
\hline $\mathrm{CH}_{4}$ & 0 & 0 & \multicolumn{1}{c}{0} & $\mathrm{CHF}_{2} \mathrm{Cl}$ & 6.856 & 7.003 & 0.147 \\
$\mathrm{CH}_{3} \mathrm{~F}$ & 2.713 & 2.530 & -0.183 & $\mathrm{CHFCl}_{2}$ & 5.528 & 6.372 & 0.844 \\
$\mathrm{CH}_{3} \mathrm{Cl}$ & 1.586 & 2.128 & 0.542 & $\mathrm{CHCl}_{3}$ & 4.316 & 5.708 & 1.392 \\
$\mathrm{CH}_{3} \mathrm{Br}$ & 1.212 & 2.014 & 0.802 & $\mathrm{CHBr}_{3}$ & 3.147 & 5.089 & 1.942 \\
$\mathrm{CH}_{3} \mathrm{I}$ & 0.586 & 1.769 & 1.183 & $\mathrm{CF}_{4}$ & 11.054 & 10.022 & -1.032 \\
$\mathrm{CH}_{2} \mathrm{~F}_{2}$ & 5.502 & 5.053 & -0.450 & $\mathrm{CF}_{3} \mathrm{Cl}$ & 9.406 & 9.353 & -0.053 \\
$\mathrm{CH}_{2} \mathrm{FCl}$ & 4.192 & 4.575 & 0.383 & $\mathrm{CF}_{3} \mathrm{Br}$ & 8.926 & 9.117 & 0.191 \\
$\mathrm{CH}_{2} \mathrm{Cl}$ & 2.966 & 4.003 & 1.037 & $\mathrm{CF}_{3} \mathrm{I}$ & 8.156 & 8.723 & 0.567 \\
$\mathrm{CH}_{2} \mathrm{ClBr}$ & 2.612 & 3.837 & 1.226 & $\mathrm{CF}_{2} \mathrm{Cl}_{2}$ & 8.026 & 8.692 & 0.666 \\
$\mathrm{CH}_{2} \mathrm{ClI}$ & 1.852 & 3.540 & 1.688 & $\mathrm{CFCl}_{3}$ & 6.636 & 8.020 & 1.384 \\
$\mathrm{CH}_{2} \mathrm{Br}$ & 2.257 & 3.662 & 1.405 & $\mathrm{CFBr}_{3}$ & 5.432 & 7.397 & 1.965 \\
$\mathrm{CH}_{2} \mathrm{BrI}$ & 1.664 & 3.373 & 1.709 & $\mathrm{CCl}_{4}$ & 5.486 & 7.343 & 1.857 \\
$\mathrm{CH}_{2} \mathrm{I}_{2}$ & 1.139 & 3.087 & 1.948 & $\mathrm{CBr}_{4}$ & 3.926 & 6.537 & 2.611 \\
$\mathrm{CHF}_{3}$ & 8.315 & 7.565 & -0.750 & & & &
\end{tabular}

TABLE 4: Coeffiients of the Quadratic Correlation of $V^{\text {EKT }}$ with the Number of Halogens $(\mathrm{eV})$

\begin{tabular}{lllll}
\hline & \multicolumn{1}{c}{$\mathrm{F}$} & \multicolumn{1}{c}{$\mathrm{Cl}$} & \multicolumn{1}{c}{$\mathrm{Br}$} & $\mathrm{I}$ \\
\hline$V_{\mathrm{X}}$ & 2.59 & 2.07 & 1.92 & 1.64 \\
$W_{\mathrm{X}}$ & -0.029 & -0.081 & -0.096 & -0.105
\end{tabular}

Values of $\Delta R$ can be obtained from the relationship $\Delta R=$ $\Delta V^{\mathrm{EKT}}-\Delta I$, using either the experimental or theoretical values of $\Delta I$. We have used the vertical experimental values here. Table 3 gives the values of $\Delta I, \Delta V^{\mathrm{EKT}}$, and $\Delta R$ obtained in this way.

A. The Systematic Variation of $\Delta V^{E K T}$. Values of $\Delta V^{\mathrm{EKT}}$ have been calculated for the 53 halomethanes having 0,1 , and 2 different halogens. To a first approximation these vary linearly with the number of halogens. A linear regression of $\Delta V^{\text {EKT }}$ versus halogen number gives $R^{2}=0.995$ with an rms deviation of $0.2 \mathrm{eV}$. As one might expect, the coefficient decreases as the electronegativity of the halogen decreases. Although this is a reasonable description, the rms deviation is not small, and in detail it is necessary to take into account nonlinear effects. For this we use an expression similar to eq 1 :

$$
\begin{aligned}
\Delta V^{\mathrm{EKT}}=n_{\mathrm{X}} V_{\mathrm{X}}+n_{\mathrm{Y}} V_{\mathrm{Y}}+\frac{n_{\mathrm{X}}\left(n_{\mathrm{X}}-1\right)}{2} W_{\mathrm{XX}}+ \\
\frac{n_{\mathrm{Y}}\left(n_{\mathrm{Y}}-1\right)}{2} W_{\mathrm{YY}}+n_{\mathrm{X}} n_{\mathrm{Y}} W_{\mathrm{XY}}
\end{aligned}
$$

where $n_{\mathrm{X}}$ and $n_{\mathrm{Y}}$ are the numbers of halogens $\mathrm{X}$ and $\mathrm{Y}$.

Upon fitting the values of $\Delta V^{\mathrm{EKT}}$ with this equation, we find that it gives a good description of the results, with $R^{2}$ equal to 0.9996 and an rms deviation of $0.05 \mathrm{eV}$. The quadratic coefficients are all negative, and we find that $W_{\mathrm{XY}}$ is, to within $10 \%$, the average of $W_{\mathrm{XX}}$ and $W_{\mathrm{YY}}$. With this approximation, eq 2 can be simplified to give

$$
\Delta V^{\mathrm{EKT}}=\sum_{\mathrm{X}} n_{\mathrm{X}}\left[V_{\mathrm{X}}+(n-1) W_{\mathrm{X}}\right]
$$

where $n$ is the total number of halogen atoms in the molecule and $W_{\mathrm{X}}=W_{\mathrm{XX}} / 2$. This expression fits the data equally as well as does eq 2. The coefficients obtained from this fit are listed in Table 4; from the fitting procedure, we find that the uncertainty is about $0.03 \mathrm{eV}$ in the $V_{\mathrm{X}}$ values and about 0.008 $\mathrm{eV}$ in the $W_{\mathrm{X}}$ values.

As expected, $V_{\mathrm{X}}$ increases with the electronegativity of the halogen. However, in contrast to the situation for $A_{\mathrm{X}}$, the increase is not linear with electronegativity. This difference is illustrated in Figure 3, where the values of $V_{\mathrm{X}}$ have been plotted as open squares connected by dashed lines. 


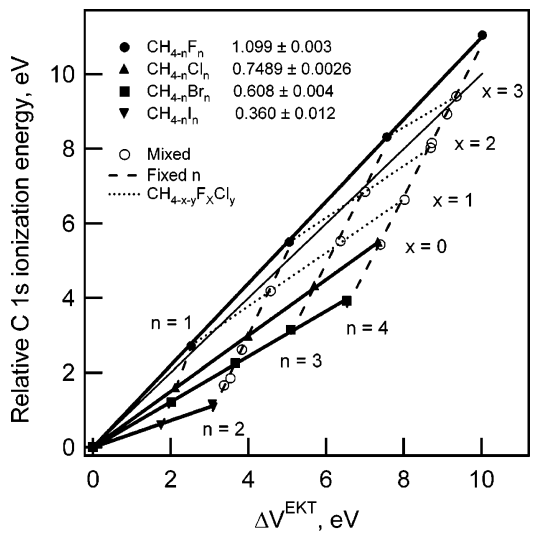

Figure 4. Experimental carbon $1 \mathrm{~s}$ ionization energy of halogenated methanes plotted against $\Delta V^{\mathrm{EKT}}$, relative to the values for methane. The thin solid line has unit slope. The other lines are least-squares fits to different families of compounds.

B. The Relationship between $\Delta V^{E K T}$ and the Ionization Energy. The shifts in ionization energy and the shifts in $V^{\text {EKT }}$ are connected through the relaxation energy: $\Delta I=\Delta V^{\mathrm{EKT}}-$ $\Delta R$. We can visualize this relationship by plotting the experimental values of $\Delta I$ versus $\Delta V^{\mathrm{EKT}}$ as in Figure 4. In this figure, each molecule is represented by a point. Each of the groups $\mathrm{CH}_{4-n} \mathrm{X}_{n}$ (with only a single halogen substituent) are indicated by special symbols, while the mixed compounds $\mathrm{CH}_{4-n-m} \mathrm{X}_{n} \mathrm{X}_{m}^{\prime}$ are indicated by open circles. The thin solid line is the locus of $\Delta I=\Delta V^{\mathrm{EKT}}$, corresponding to $\Delta R=0$. The values of $\Delta R$ are, therefore, the vertical distances between this line and the points. Except for the fluorinated compounds, all of the data fall below this line, indicating that the relaxation energy is, in most cases, greater than it is in methane. This result is not surprising, in that the relaxation energy reflects the polarizability of the substituents, which is greater for chlorine, bromine, and iodine than for hydrogen. The points for fluorine fall above the line, indicating that fluorine has a lower polarizability than hydrogen.

The striking and unexpected feature of this figure is the number of linear relationships that are apparent in it. The heavy solid lines, the dashed lines, and the dotted lines represent leastsquares fits of straight lines to the data for particular families of compounds. For instance, the heavy lines are fits to the data for the compounds with only a single halogen, $\mathrm{CH}_{4-n} \mathrm{X}_{n}$. They fit the data quite well, indicating that for these compounds $\Delta I$ is directly proportional to $\Delta V^{\mathrm{EKT}}$. There is no indication of any nonlinearity. The dashed lines show fits for molecules each of which has the same number, $n$, of halogen atoms, $\mathrm{CH}_{4-n} \mathrm{X}_{n-m} \mathrm{X}_{m}^{\prime}$, and the dotted lines show the fits for the fluorochloromethanes, $\mathrm{CH}_{4-x-y} \mathrm{~F}_{x} \mathrm{Cl}_{y}$ for 1,2 , and 3 fluorine atoms. It is apparent that these data are well described by linear relationships.

Because of the relationship between $\Delta I, \Delta V^{\mathrm{EKT}}$, and $\Delta R$, it follows that if $\Delta I$ is proportional to $\Delta V^{\mathrm{EKT}}$, then also $\Delta R$ must also be proportional to $\Delta V^{\mathrm{EKT}}$. Thus, for the compounds containing only one kind of halogen, we can write

$$
\Delta I_{\mathrm{X}, n}=\Delta V_{\mathrm{X}, n}^{\mathrm{EKT}}-\Delta R_{\mathrm{X}, n}=\Delta V_{\mathrm{X}, n}^{\mathrm{EKT}}\left(1-r_{\mathrm{X}}\right)=\Delta V_{\mathrm{X}, n}^{\mathrm{EKT}} F_{\mathrm{X}}
$$

where $\Delta I_{\mathrm{X}, n}$ is the shift in ionization energy associated with $n$ halogen atoms of type $\mathrm{X}$ and $\Delta V_{\mathrm{X}, n}^{\mathrm{EKT}}$ and $\Delta R_{\mathrm{X}, n}$ are the corresponding shifts in $V^{\mathrm{EKT}}$ and $R$. The quantity $r_{\mathrm{X}}$ is the proportionality constant between $\Delta R$ and $\Delta V^{\mathrm{EKT}}$, and $F_{\mathrm{X}}$ is the slope of the appropriate line in Figure 4. These slopes are indicated in the figure. The significant result here is that a single relaxation parameter is sufficient to describe the relaxation effect of each halogen, no matter how many atoms of that halogen are present.

While eq 4 provides a good description of the compounds with only one type of halogen, it does not deal with the mixed compounds. As a hypothesis, we consider the possibility that the relaxation energy associated with each halogen is independent of what other halogens are present. However, mathematical expressions based on this idea do not give a good description of the relationship between $\Delta I$ and $\Delta V^{\mathrm{EKT}}$. It is necessary to take into account that the effect of one halogen is modified by the presence of other halogens. A good description is obtained if $r_{\mathrm{X}}$ in eq 4 is replaced by a weighted average $\sum_{n} n_{\mathrm{X}} r_{\mathrm{X}} / n$, where $n_{\mathrm{X}}$ is the number of halogen atoms of type $\mathrm{X}$ and $n$ the total number of halogen atoms. Then we have

$$
\Delta I=\Delta V^{\mathrm{EKT}} \sum_{\mathrm{X}} F_{\mathrm{X}} n_{\mathrm{X}} / n
$$

with only one parameter for each halogen. This expression describes the relationship between $\Delta I$ and $\Delta V_{\text {EKT }}$ quite well $\left(R^{2}\right.$ $=0.9997$ with an rms deviation of $0.055 \mathrm{eV}$ ). The parameters derived from this fit are similar to those shown in Figure 4: $F_{\mathrm{F}}$ $=1.101 \pm 0.003, F_{\mathrm{Cl}}=0.744 \pm 0.004, F_{\mathrm{Br}}=0.610 \pm 0.005$, and $F_{\mathrm{I}}=0.370 \pm 0.012$.

The corresponding values of $r_{\mathrm{X}}$, equal to $1-F_{\mathrm{X}}$, reflect the difference between hydrogen and the halogens in their contributions to the relaxation. Since these arise because of the polarization of the electrons on the substituent in response to the formation of the core hole, they may be expected to depend on the difference in polarizability of the halogen relative to that of hydrogen. The values of $r_{\mathrm{X}}$ increase monotonically from -0.101 for fluorine to +0.630 for iodine, reflecting the increase in polarizability of the halogen with increasing size.

\section{Discussion}

The systematic behavior described above can be summarized as follows.

(1) The ionization-energy shifts, $\Delta I$, vary approximately linearly with the number of each kind of halogen. A better description is obtained if quadratic terms in the halogen numbers are included, as in eq 1.

(2) The linear coefficients are proportional to the difference in electronegativity between the halogen and hydrogen, as illustrated in Figure 3. Also $\Delta I$ varies nearly linearly with the sum of the electronegativities of the halogens (Figure 2). It is not readily apparent why there should be such simple relationships between these quantities.

(3) The initial-state contribution, $\Delta V^{\mathrm{EKT}}$, to the ionizationenergy shift is approximately linear in halogen number, but a much better description is obtained if a quadratic expression is used. In contrast to the situation for $\Delta I$, the linear coefficients, though they increase monotonically with electronegativity, are not proportional to the electronegativity difference.

(4) $\Delta I$ and $\Delta V^{\text {EKT }}$ are related by a multiplier that reflects the weighted relaxation contributions of all of the halogen substituents, as indicated by eq 5 .

(5) The relaxation contributions increase with the size and polarizability of the halogens.

$\Delta \boldsymbol{V}^{\mathbf{E K T}}$ and $\boldsymbol{V}_{\mathrm{X}}$. We begin the discussion of these observations with $\Delta V^{\mathrm{EKT}}$ and $V_{\mathrm{X}}$. These should be the simplest quantities to understand, in that they depend only on ground-state properties (primarily the charge distribution) of the un-ionized 
molecules. Furthermore, understanding these quantities is fundamental to understanding the others.

We gain insight into $V_{\mathrm{X}}$ by considering a simple point-charge model, bearing in mind that such a model can give only a rough picture of the system. In terms of this model, $V_{\mathrm{X}}$, which is the change in $V^{\mathrm{EKT}}$ when one hydrogen in methane is replaced by halogen $\mathrm{X}$, is given by the expression

$$
V_{\mathrm{X}}=-\sum_{\mathrm{i}} q_{\mathrm{i}}\left(k_{\mathrm{C}}-1 / R_{\mathrm{i}}\right)+\sum_{\mathrm{i}} q_{\mathrm{i}}^{\prime}\left(k_{\mathrm{C}}-1 / R_{\mathrm{i}}^{\prime}\right)
$$

where $q_{\mathrm{i}}$ is the charge on ligand $\mathrm{i}$ and $R_{\mathrm{i}}$ is the distance between ligand $\mathrm{i}$ and the carbon atom. The sum is taken over all four ligands. The unprimed quantities refer to the molecule of interest and the primed quantities to the reference molecule, methane. The quantity $k_{\mathrm{C}}$ is the change in $V$ when a valence electron is removed. For removal of a $2 p$ electron from atomic carbon, atomic structure calculations indicate that this should be about 0.5 au. ${ }^{35,36}$ For removal of a 2 s electron it will be somewhat greater. Since the carbon-hydrogen bond length is about 2 au, the terms in eq 6 that reflect the contribution due to the hydrogen ligands are small and can, to a reasonable approximation, be ignored. Then we have

$$
V_{\mathrm{X}}=-q_{\mathrm{X}}\left(k_{\mathrm{C}}-1 / R_{\mathrm{X}}\right)
$$

The important features to note here are that $V_{\mathrm{X}}$ is approximately proportional to the charge on the halogen and that the proportionality constant depends on the difference between $k_{\mathrm{C}}$ and $1 / R_{\mathrm{X}}$. For fluorine, $R_{\mathrm{F}}$ is relatively small and $1 / R_{\mathrm{F}}$ is correspondingly large. In consequence, the proportionality constant for fluorine is small. By contrast, $R_{\mathrm{I}}$ is large and $1 / R_{\mathrm{I}}$ correspondingly small, with the consequence that the proportionality constant for iodine is large. The sensitivity of $V$ to the amount of charge withdrawn is therefore much greater for iodine than for fluorine. This accounts for the nonlinear dependence of $V_{\mathrm{X}}$ on electronegativity seen in Figure 3. A small amount of charge transfer for iodomethane produces a relatively large effect, whereas a much larger amount of charge transfer for fluoromethane does not produce a proportionately larger effect.

Using eq 7 and assuming a value of $k_{\mathrm{C}}$ of $0.5 \mathrm{au}$, we can obtain values of $q_{\mathrm{X}}$ from the values of $V_{\mathrm{X}}$. These range from -0.24 for iodomethane to -0.79 for fluoromethane and are, to a good approximation, proportional to the electronegativity difference between the halogen and hydrogen. The proportionality constant is 0.43 . Such proportionality is more or less expected. Gordy ${ }^{37}$ proposed that the ionic character of a bond was equal to half the electronegativity difference, and our result is consistent with this. Application of the principle of electronegativity equalization, using parameters given by Bergmann and Hinze, ${ }^{28}$ also leads to halogen charges proportional to the electronegativity difference (although the charges estimated in this way are only about $1 / 3$ of those obtained by the analysis given above).

Thus, our picture of the initial-state effect is that the charge withdrawn from carbon by the halogen is proportional to the electronegativity difference between the halogen and hydrogen. However, the sensitivity of $V^{\mathrm{EKT}}$ to the charge increases with the size of the halogen, with the result that $V_{X}$ increases nonlinearly with the electronegativity difference.

The quadratic term in eq 3 has a simple interpretation. Each halogen atom, $\mathrm{X}$, withdraws electrons from the central carbon and, in so doing, it increases $V_{\text {EKT }}$ by an amount $V_{\mathrm{X}}$. However, the positive charge that is produced at the carbon atom by this halogen polarizes all of the $n$ halogens, and this polarization

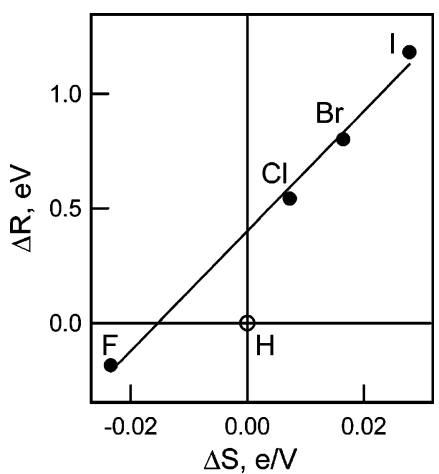

Figure 5. $\Delta R$ for $\mathrm{CH}_{3} \mathrm{X}$ plotted against the relative softness of the halogen.

reduces $V_{\text {EKT. }}$ It is to be noted in Table 4 that $W_{\mathrm{X}}$ increases in magnitude with the size of the halogen (and, hence, with its polarizability). Thus, both $V_{\mathrm{X}}$ and $W_{\mathrm{X}}$ are affected by the size of the halogen. This reduction of the effect of an electronegative substituent by the addition of a polarizable substituent has been noted in other contexts ${ }^{38}$ and is responsible for the higher acidity of formic acid relative to acetic acid. ${ }^{39}$

$\Delta \boldsymbol{R}$. The relaxation energy arises from the polarization of the surroundings by the core hole. We can approximately separate this polarization into three parts: shrinkage of the carbon atomic electrons toward the core hole, transfer of electrons from the ligands to the core-ionized atom, and polarization of electrons on the ligand. To a first approximation, the first of these is more or less independent of the surroundings, although it is to be noted that there will be fewer carbon electrons on $\mathrm{CF}_{4}$ than on $\mathrm{CH}_{4}$ to participate in this process. The second and third can be regarded as different degrees of polarization of the ligand, and we may anticipate that the relaxation energies will be related to the polarizability of the ligands. This is, in turn, related to the hardness of the ligand, $\eta$, since it has been shown that the reciprocal of the hardness is proportional to the polarizability. ${ }^{2,3}$ To illustrate the relationship between the relaxation energy and the hardness, we have plotted in Figure 5 the relaxation energies for $\mathrm{CH}_{3} \mathrm{X}$ versus the softness $^{40}\left(S=(2 \eta)^{-1}\right)$ of $\mathrm{X}$ (relative to that of hydrogen). (Values of hardness have been taken from Bergmann and Hinze. ${ }^{28}$ ) We see that there is a nearly linear relationship between the relaxation energy for the halomethanes and the softness of the corresponding halogen.

For the compounds containing more than one kind of halogen, we have seen that the relaxation energy is not a simple sum of independent contributions from the individual halogens, but a weighted average. We can understand this by considering, for instance, the charges on the halogens in $\mathrm{CCl}_{4}, \mathrm{CF}_{2} \mathrm{Cl}_{2}$, and $\mathrm{CF}_{4}$. The charges have been determined by using electronegativity equalization and the parameters given by Bergmann and Hinze. ${ }^{28}$ While these may not accurately represent the actual charges, they suffice to illustrate the principles involved. In $\mathrm{CCl}_{4}$ the chlorine charge is -0.035 whereas in $\mathrm{CCl}_{2} \mathrm{~F}_{2}$ it is +0.036 ; the fluorine atoms have taken charge from the chlorine atoms. In $\mathrm{CCl}_{2} \mathrm{~F}_{2}$ the fluorine charge is -0.15 , whereas in $\mathrm{CF}_{4}$ it is -0.09 . Thus, in $\mathrm{CCl}_{2} \mathrm{~F}_{2}$ chlorine can contribute less per atom to the relaxation than it does in $\mathrm{CCl}_{4}$, while fluorine can contribute more per atom than it does in $\mathrm{CF}_{4}$. The averaging process used in eq 6 provides a way to include this effect.

$\Delta \boldsymbol{I}$. We can approximately understand the linear relationship between carbon 1s ionization energy and electronegativity by reference to Figure 3, where the linear constants $A_{\mathrm{X}}$ and $V_{\mathrm{X}}$ are 
plotted against electronegativity. As has been noted, $A_{\mathrm{X}}$ is the change in ionization energy when one hydrogen in methane is replaced by halogen $\mathrm{X}$. It is observed to be proportional to the difference in electronegativity of the halogen and that of hydrogen. $V_{\mathrm{X}}$ is the difference in $V^{\mathrm{EKT}}$ for the same substitution. Although it varies monotonically with the electronegativity difference, it is by no means proportional to this difference.

As we have seen, the values of $V_{X}$ can be understood in terms of a charge transfer to the halogen that is proportional to the electronegativity difference. The effect of this charge on the ionization energy depends on the distance between the carbonhalogen bond length. For a given charge transfer, there is a relatively large positive effect for iodine and a relatively small positive effect for fluorine, in keeping with the different bond lengths. However, since the charge transfer is much greater for fluorine than for iodine, the overall effect on the ionization energy is greater for fluorine.

In addition to this initial-state effect, each of the halogens produces a relaxation effect that modifies the ionization energy shift. For iodine, because of its high polarizability, the relaxation is large and negative, and for fluorine it is small and positive. The result of the relaxation is to move the value of $\Delta I$ for iodomethane from the point indicated by the open marker in Figure 3 down to the point indicated by the solid marker. The points for the other halogens are moved in a similar way. The result is the linear relationship that is observed. It is to be emphasized, however, that there is no apparent reason this should end up with a linear relationship. All we can say is that the relaxation energies are of the correct sign and relative magnitudes to shift the ionization energies in this direction. It still remains unclear why the relationship should be so closely linear.

An Overview. We see that the observed ionization energies result from the combined effects of the electronegativity of the halogens, the size of the halogens, and the hardness of the halogens. When a hydrogen atom is replaced by a halogen, there is transfer of negative charge to the halogen in proportion to its electronegativity. The effect of a given charge transfer on the ionization energy is influenced by the size of the halogen, being relatively more positive as the size of the halogen increases. Opposing this, the ionization energy is shifted negatively by the relaxation in proportion to the polarizability of the halogen (inverse hardness). As a result, the effect of bond length on $V^{\text {EKT }}$ is essentially offset by the effect of polarizability on the relaxation energy.

The three quantities - electronegativity, hardness, and atomic size-are not independent, but are all reflections of the same phenomenon: How strongly does a halogen attract electrons? Strong attraction leads to high electronegativity and hardness and to small size; it has been observed that electronegativity and hardness are nearly proportional to one another. ${ }^{3,41,42}$ The close relationship between these three quantities for the halogens is illustrated in Figure 6, where we have plotted the softness of the halogen (left ordinate) and carbon-halogen bond length (right ordinate) versus electronegativity. It is apparent that the softness and bond length track closely with each other and both track with the electronegativity. Where there is a large step between fluorine and chlorine in electronegativity, there is also a large step in the other two quantities.

What are the consequences of these observations for using inner-shell ionization energies to determine group electronegativities. True et al. ${ }^{25}$ suggested that measurements of the carbon $1 \mathrm{~s}$ ionization energies of $\mathrm{CH}_{3} \mathrm{R}$ could be used with the relationship shown in Figure 2 to determine the electronegativity

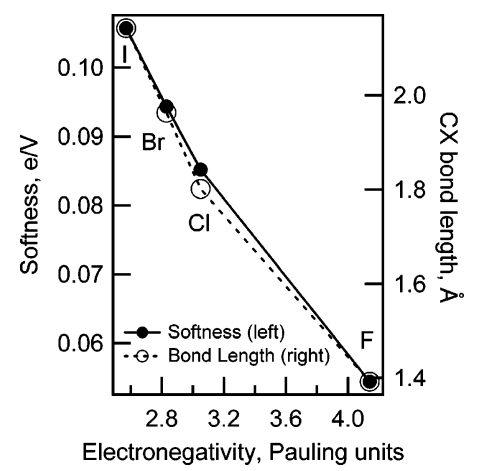

Figure 6. Softness (left axis) and carbon-halogen bond length (right axis) plotted against Pauling electronegativity.

of $R$. They showed that this procedure gave reasonable results for $\mathrm{CH}_{3}, \mathrm{NH}_{2}$, and $\mathrm{OH}$ and applied it to the determination of the electronegativities of $\mathrm{CF}_{3}$ and $\mathrm{SF}_{5}$. We now see that the linear correlation seen in Figure 2 results from an interplay of the correlated quantities electronegativity, hardness, and bond length. It is by no means clear that the same correlations will exist for substituent groups, and there is evidence that this is not the case. As was noted by True et al. the calculated hardness for a large number of substituent groups ${ }^{32}$ shows little correlation with electronegativity. Or, if we compare the effects of, for instance, the ethyl, ethenyl, and ethynyl groups, we expect to find very different electronegativities, ${ }^{32}$ but the effective bond lengths are similar. Thus, extension of this procedure to the determination of group electronegativities must be done with caution.

Acknowledgment. We are pleased to acknowledge support from the Divisions of Chemical and Material Sciences, Office of Energy Research, of the U.S. Department of Energy, the Research Council of Norway (NFR), and the EC Access to research Infrastructure Program (ARI) and the Nordic Academy of Advanced Study.

\section{References and Notes}

(1) Parr, R. G.; Pearson, R. G. J. Am. Chem. Soc. 1983, 105, 7512

(2) Politzer, P. J. Chem. Phys. 1987, 86, 1072-1073.

(3) Politzer, P.; Huheey, J. E.; Murray, J. S.; Grodzicki, M. J. Mol. Struct. (THEOCHEM) 1992, 259, 99.

(4) http://www-als.lbl.gov/als/techspecs/bl10.0.1.html.

(5) http://www.maxlab.lu.se/.

(6) Carroll, T. X.; Bozek, J. D.; Kukk, E.; Myrseth, V.; Saethre, L. J.; Thomas, T. D.; Wiesner, K. J. Electron Spectrosc. Relat. Phenom. 2002, $125,127$.

(7) Myrseth, V.; Bozek, J. D.; Kukk, E.; Saethre, L. J.; Thomas, T. D. J. Electron Spectrosc. Relat. Phenom. 2002, 122, 57.

(8) SPANCF, http://www.geocities.com/ekukk 8,35 .

(9) Van der Straten, P.; Morgenstern, R.; Niehaus, A. Z. Phys. D 1988

(10) Carroll, T. X.; Børve, K. J.; Saethre, L. J.; Bozek, J. D.; Kukk, E.; Hahne, J. A.; Thomas, T. D. J. Chem. Phys. 2002, 116, 10221.

(11) Carroll, T. X.; Hahne, J.; Thomas, T. D.; Saethre, L. J.; Berrah, N.; Bozek, J.; Kukk, E. Phys. Rev. A 2000, 61, 042503.

(12) Sundin, S.; Saethre, L. J.; Sorensen, S. L.; Ausmees, A.; Svensson, S. J. Chem. Phys. 1999, 110, 5806.

(13) Jolly, W. L.; Bomben, K. D.; Eyermann, C. J. At. Data Nucl. Data Tables 1984, 31, 433.

(14) Holmes, S. A. MS thesis, Oregon State University, 1974. The reference compound for these measurements was $\mathrm{CF}_{4}$. The values given by Holmes and appearing in ref 13 have been reduced by $0.06 \mathrm{eV}$ to reflect the latest value for the $\mathrm{C} 1 \mathrm{~s}$ ionization energy of $\mathrm{CF}_{4}$ (ref 7).

(15) Jolly, W. L.; Bakke, A. A. J. Am. Chem. Soc. 1976, 98, 6500.

(16) Perry, W. B.; Jolly, W. L. Inorg. Chem. 1974, 13, 1211. The carbon 1s ionization energies given by Perry and Jolly are lower than those obtained in more recent measurements by an average of $0.13 \mathrm{eV}$. Their reported value for $\mathrm{CBr}_{4}$ has, therefore, been increased by this amount.

(17) Frisch, M. J.; Trucks, G. W.; Schlegel, H. B.; Scuseria, G. E.; Robb, M. A.; Cheeseman, J. R.; Zakrzewski, V. G.; Montgomery, J. A., Jr.; 
Stratmann, R. E.; Burant, J. C.; Dapprich, S.; Millam, J. M.; Daniels, A. D.; Kudin, K. N.; Strain, M. C.; Farkas, O.; Tomasi, J.; Barone, V.; Cossi, M.; Cammi, R.; Mennucci, B.; Pomelli, C.; Adamo, C.; Clifford, S.; Ochterski, J.; Petersson, G. A.; Ayala, P. Y.; Cui, Q.; Morokuma, K.; Malick, D. K.; Rabuck, A. D.; Raghavachari, K.; Foresman, J. B.; Cioslowski, J.; Ortiz, J. V.; Baboul, A. G.; Stefanov, B. B.; Liu, G.; Liashenko, A.; Piskorz, P.; Komaromi, I.; Gomperts, R.; Martin, R. L.; Fox, D. J.; Keith, T.; AlLaham, M. A.; Peng, C. Y.; Nanayakkara, A.; Gonzalez, C.; Challacombe, M.; Gill, P. M. W.; Johnson, B.; Chen, W.; Wong, M. W.; Andres, J. L.; Gonzalez, C.; Head-Gordon, M.; Replogle, E. S.; Pople, J. A. Gaussian 98; Gaussian, Inc.: Pittsburgh, PA, 1998.

(18) Martin, J. M. L.; Sundermann, A. J. Chem. Phys. 2001, 114, 3408. (19) Bergner, A.; Dolg, M.; Kuechle, W.; Stoll, H.; Preuss, H. Mol. Phys. 1993, 80, 1431.

(20) http://www.emsl.pnl.gov/forms/basisform.html.

(21) Stevens, W. J.; Basch, H.; Krauss, M. J. Chem. Phys. 1984, 81, 6026.

(22) Karlsen, T.; Børve, K. J. J. Chem. Phys. 2000, 112, 7979

(23) Allred, A. L. J. Inorg. Nucl. Chem. 1961, 17, 215.

(24) Thomas, T. D. J. Am. Chem. Soc. 1970, 92, 4184.

(25) True, J. E.; Thomas, T. D.; Winter, R. W.; Gard, G. L. Inorg. Chem. 2003, 42, 4437.

(26) Gordy, W.; Thomas, W. J. O. J. Phys. Chem. 1956, 24, 439.

(27) Wells, P. R. Prog. Phys. Org. Chem. 1968, 6, 111.
(28) Bergmann, D.; Hinze, J. Angew. Chem., Int. Ed. Engl. 1996, 35,

(29) Sanderson, R. T. Chemical Periodicity; Rheinhold Publishing Co.: New York, 1960; p 32

(30) Mulliken, R. S. J. Chem. Phys. 1934, $2,782$.

(31) Allen, L. C. J. Am. Chem. Soc. 1989, 111, 9003.

(32) Bergmann, D.; Hinze, J. In Structure and Bonding, No. 66; Sen, K. D., Jørgensen, C. K., Eds.; Springer-Verlag: Berlin, Germany, 1987; p 145.

(33) Allred, A. L.; Rochow, E. G. J. Inorg. Nucl. Chem. 1958, 5, 264. (34) Børve, K. J.; Thomas, T. D. J. Electron Spectrosc. Relat. Phenom. 2000, 107, 155 .

(35) Snyder, L. C. J. Chem. Phys. 1971, 55, 95.

(36) Clementi, E.; Roetti, C. At. Data Nucl. Data Tables 1974, 14, 177.

(37) Gordy, W. Discuss. Faraday Soc. 1955, 19, 14.

(38) Siggel, M. R. F.; Thomas, T. D. J. Electron Spectrosc. Relat. Phenom. 1989, 48, 101

(39) Siggel, M. R. F.; Thomas, T. D. J. Am. Chem. Soc. 1992, 114, 5795.

(40) Chattaraj, P. K.; Parr, R. G. In Structure and Bonding, No. 80;

Sen, K. D., Ed.; Springer-Verlag: Berlin, Germany, 1993; pp 11-25.

(41) Nalewajski, R. F. J. Phys. Chem. 1985, 89, 2831.

(42) Yang, W.; Lee, C.; Ghosh, S. K. J. Phys. Chem. 1985, 89, 5412. 